\title{
Identification of key candidate genes and pathways in hepatocellular carcinoma by integrated bioinformatical analysis
}

\author{
TONGHAI XING ${ }^{1}$, TINGMANG YAN ${ }^{2}$ and QIANG ZHOU ${ }^{1}$ \\ Departments of ${ }^{1}$ General Surgery and ${ }^{2}$ Urology, Shanghai General Hospital, School of Medicine, \\ Shanghai Jiao Tong University, Shanghai 200080, P.R. China
}

Received October 30, 2017; Accepted March 13, 2018

DOI: $10.3892 /$ etm.2018.6075

\begin{abstract}
Hepatocellular carcinoma (HCC) is one of the most common malignant neoplasms worldwide, however the underlying mechanisms and gene signatures of HCC are unknown. In the present study the profile datasets of four cohorts were integrated to elucidate the pathways and candidate genes of HCC. The expression profiles GSE25097, GSE45267, GSE57957 and GSE62232 were downloaded from the Gene Expression Omnibus database, including 436 HCC and 94 normal liver tissues. A total of 185 differentially expressed genes (DEGs) were identified in HCC, including 92 upregulated genes and 92 downregulated genes. Gene ontology (GO) was performed, which revealed that the upregulated DEGs were primarily enriched in cell division, mitotic nuclear division, mitotic cytokinesis and G1/S transition of the mitotic cell cycle. Pathway enrichment was analyzed based on the Kyoto Encyclopedia of Genes and Genomes database to assess the functional relevance of DEGs. The most significant module was selected from protein-protein interactions and 15 important hub genes were identified. The sub-networks of hub genes were involved in cell division, p53 signaling, and $\mathrm{T}$ lymphotropic virus type I infection signaling pathways. In conclusion, the present study revealed that the identified DEG candidate genes may promote the understanding of the cause and molecular mechanisms underlying the development of $\mathrm{HCC}$ and that these candidates and signal pathways may be potential targets of clinical therapy for HCC.
\end{abstract}

\section{Introduction}

Hepatocellular carcinoma (HCC) is one of the most common malignant neoplasms worldwide (1). Its incidence is particularly

Correspondence to: Dr Qiang Zhou, Department of General Surgery, Shanghai General Hospital, School of Medicine, Shanghai Jiao Tong University, 100 Haining Road, Shanghai 200080, P.R. China

E-mail: zqzhouqiang@sjtu.edu.cn

Key words: hepatocellular carcinoma, bioinformatical analysis, differentially expressed genes high in China (2) on account of hepatitis B (HBV) and C (HCV) infection. However, in the western world, HCC is primarily due to non-alcoholic fatty liver disease (NAFLD) $(3,4)$. The aggressive nature of this malignancy combined with the limited therapeutic options result in poor prognosis $(1,5)$. Unfortunately, $\mathrm{HCC}$ has a high recurrence rate even with successful surgical removal because of the insensitivity of HCC to chemotherapy agents and radiotherapy (6). Although there have been extensive previous studies on the molecular mechanism of HCC formation and progression, the causes of HCC are not yet clear. Hence, owing to the high morbidity and mortality in HCC, it is very important to determine the underlying molecular mechanisms and pathological processes and identify suitable molecular biomarkers for early HCC diagnosis.

While HCC is a highly heterogeneous tumor, with different genetic compositions and multifaceted molecular pathogenesis $(7,8)$, it is also a slow process during which plenty of genomic alterations accumulate and change the hepatocellular phenotype, leading to cellular intermediates and multiple monoclonal that evolve into $\operatorname{HCC}(9,10)$. Microarray technology has become an indispensable tool to monitor genome wide expression levels of genes in a given organism. Gene Expression Omnibus (GEO) and The Cancer Genome Atlas (TCGA) are two public functional genomics data repositories accepting data from diverse microarray platforms (11). Nowadays, gene expression studies by microarray have been performed to uncover molecular variations in HCC (12-14). Pathway analysis has shown several important cellular signaling pathway alterations to be linked with the main pathogenic mechanism (8). A small number of hub genes (proteins) were identified as key modulators in HCC metastasis by using protein-protein interaction (PPI) network. However, the most significantly dysregulated genes from previous studies are inconsistent because of the sample heterogeneity in independent studies, small sample size, and different statistical methods.

In the present study, four original microarray datasets GSE25097, GSE45267, GSE57957, and GSE62232 were downloaded from the NCBI-Gene Expression Omnibus database (NCBI-GEO) (available online: https://www.ncbi.nlm.nih.gov/geo/), and included 436 HCC and 94 normal liver tissues. The microarray data was normalized and preprocessed using the robust multi-array average algorithm (RMA). The differentially expressed genes (DEGs) were obtained using the limma package. Venny was applied to 
filter the overlapping DEGs among groups. Gene ontology (GO) and pathway enrichment analysis were also performed for annotation and visualization withDAVID and KEGGPATHWAY (Available online: http://www.genome.jp/kegg). We used the STRING online database (Available online: http://string-db.org) to annotate functional interactions of DEGs. The most significant module was selected from PPI, and 15 important hub genes were identified. The sub-networks of hub genes were involved in cell division, p53 signaling and HTLV-I infection signaling pathways.

\section{Materials and methods}

Microarray data information and data preprocessing. The microarray data from GSE25097, GSE45267, GSE57957, and GSE62232 were downloaded from the GEO database (http://www.ncbi.nlm.nih.gov/geo/). The microarray data of GSE25097 was based on GPL10687 platforms (Rosetta/Merck Human RSTA Affymetrix 1.0 microarray, Custom CDF; Affymetrix Inc., Santa Clara, CA, USA). The microarray data of GSE45267 was based on GPL570 platforms (HG-U133 Plus_2, Affymetrix Human Genome U133 Plus 2.0 Array). The microarray data of GSE57957 was based on GPL10558 platforms (Illumina HumanHT-12 v4.0 expression beadchip). The microarray data of GSE62232 was based on GPL570 platforms (HG-U133_Plus_2, Affymetrix Human Genome U133 Plus 2.0 Array). We chose these four datasets for integrated analysis, because they represented different racial populations. The microarray data was preprocessed using the RMA with the Affy and limma package in Bioconductor (v1.46.1; http://www.bioconductor.org/). Background correction, normalization, and calculation of expression were all included in the preprocessing stage. The microarray data probe was transformed to gene symbols with Bioconductor Annotation Data software packages. If several probes were mapped to one gene symbol, the mean value was set as the final expression value of this gene. The DEGs in every group were analyzed using the limma package. In Bioconductor. The DEG P-values were calculated using the unpaired or paired Student's t-test provided by the limma package. $\mathrm{P}<0.01$ and $[\log \mathrm{FC}]>1$ were used as cut-off criterion for DEGs. Hierarchical clustering analysis of the DEGs was then performed and visualized using g-plots in the $\mathrm{R}$ package. Venny is an interactive tool used to compare lists with Venn diagrams. The intersections of upregulated and downregulated genes in different sample groups were respectively analyzed using Venny v2.0.

GO and pathway enrichment analysis. Candidate DEG functions and pathway enrichment were analyzed using multiple online databases; among them, DAVID is a website with gene annotation, visualization, and integrated discovery function, and can thus provide gene biological meaning. GO analysis and pathway analysis were carried out using the KEGG PATHWAY (available online: http://www.genome.jp/kegg), Reactome (available online: http://www.reactome.org), and GO website, with $\mathrm{P}<0.01$ as the cut-off criterion $(15,16)$.

Integration of protein-protein interaction (PPI) network, modular analysis and significant candidate genes and pathway identification. First, online database STRING (available online: http://string-db.org) was employed to develop DEGs-encoded proteins and protein-protein interaction network (PPI). Second, the Cytoscape software (17) was utilized to construct a protein interaction relationship network and analyze the interaction relationship of the candidate DEGs encoding proteins in colon cancer. Third, the Network Analyzer plug-in was used to calculate the node degree, i.e., the numbers of inter-connections to filter the hub genes of PPI. The corresponding proteins in the central nodes might be the core proteins and key candidate genes that have important physiological regulatory functions. The cBio Cancer Genomics Portal (http://cbioportal.org) is an open platform for exploring multidimensional cancer genomics data by encapsulating molecular profiling data obtained from cancer tissue and cell lines into readily understandable genetic, epigenetic, gene expression, and proteomic events. Complex cancer genomic profiles can be easily accessed using the query interface of the portal enabling researchers to explore and compare genetic alterations across samples. The underlying data thus obtained can be linked to clinical outcomes to facilitate novel discovery in biological systems. Through use of the portal search function, identified DEG candidate genes are classified as altered or not altered. The genomics datasets are then presented using OncoPrint as heatmaps-a visually appealing display of alterations in gene arrays across tumor samples.

\section{Results}

Identification of DEGs in HCC. NCBI-GEO is a public functional genomics data repository accepting data from diverse microarray platforms, from which gene expression profiles of HCC and normal or adjacent liver tissue from the GSE25097, GSE45267, GSE57957, and GSE62232 databases were obtained. The microarray data of GSE25097 had 268 HCC tissues and 6 normal liver tissues (18); the GSE62232 data had $81 \mathrm{HCC}$ tissues and 10 normal liver tissues (12); the GSE45267 data included 48 HCC tissues and 39 healthy liver tissues; and the GSE57957 data had 39 pairs of HCC tissues and matched paraneoplastic tissue (19). Using $\mathrm{P}<0.01$ and $[\log \mathrm{FC}]>1$ as cut-off criterion, we extracted 1072, 1850,4055, and 1876 DEGs from the expression profile datasets GSE25097, GSE45267, GSE57957, and GSE62232, respectively. In total, 185 overlapping DEGs were identified in HCC (Fig. 1). However, when we determined the intersections of upregulated and downregulated genes in four sample groups using Venny, one gene was excluded because of it's contradictory expression in different groups. So, we ultimately identified 92 upregulated genes and 92 downregulated genes (Table I).

DEGs GO analysis in HCC. DEGs GO analysis was conducted with the online softwares DAVID and Gene Ontology. The DEGs were classified into three functional groups: Biological process group, molecular function group, and cellular component group (Fig. 2A). As shown in Fig. 2B and Table II, cell division, mitotic nuclear division, and G1/S transition of mitotic cell cycle are the most abundant groups in the 'biological process' category, with each accounting for about $20 \%$ of upregulated genes. In contrast, downregulated genes were mainly enriched in signal transduction and proteolysis. For the molecular function category, protein binding (71.7\%) was 
Table I. Up and downregulated DEGs.

DEGs

Genes name

Upregulated

TPX2 GPSM2 IGSF3 CCDC34 ZIC2 ANLN SMYD3 RACGAP1 NUP37 SULT1C2 AURKA ATP6V1C1 TTC13 KIF4A THY1 SQLE MCM5 TMEM106C MELK ZWINT OIP5 PTTG1 TARBP1 STMN1 UBE2T CKS2 TP53I3 SLC38A6 TBCE CCNB2 MCM7 PRC1 CEP55 CCNE2 MTHFD1L MCM2 MCM4 CNIH4 DLGAP5 RAD51AP1 RAP2A COL4A1 GPC3 CDKN2C PLVAP PIGC C8orf33 DTNA NEK2 FANCD2 RFX5 COL15A1 GSTA4 ERMP1 MCM3 PCNA CENPA CDC20 RFC4 BUB1 PBK RFXANK TRIP13 PDCD2 P4HA2 SMC2 TMEM9 ASPM CDCA3 ATAD2 PEA15 UBE2C STXBP6 MICB MDK TOP2A TUBG1 RNASEH2A CAP2 FAM83D HMMR MCM6 GMNN KIF20A SAE1 CDKN2A TTK CDKN3 E2F3 NCAPG FDPS NUSAP1

Downregulated CYP26A1 IGF1 C1RL SORL1 CYP2C19 GHR CLEC1B STARD5 SHBG ATOH8 CPEB3 CRHBP QKI DBH SRD5A2 ADRA1A PLSCR4 RCAN1 CLEC4M ESR1 GCH1 FOXO1 APOF PDE7B GREM2 TTC36 CXCL2 LYVE1 ASPG NAAA NAT2 NCOR1 GCKR OIT3 CETP SRPX MT1F KCNN2 GSTZ1 OLFML3 CNDP1 CCBE1 MASP1 FOSB MARCO LCAT RSPO3 HAMP STAB2 HGFAC SLC4A4 PZP C1QTNF1 CXCL14 MBNL2 EFHD1 IGFALS MT1M ANTXR2 ECM1 FCN2 CHST4 MAN1C1 ST3GAL6 KBTBD11 PCK1 RND3 IL1RAP TMEM27 MT1X AADAT ACADS RNF165 CYP1A2 LPA LIFR EXPH5 COLEC10 PAMR1 CXCL12 LARP1B ANGPTL6 MSRA SOCS2 SARDH LY6E FCN3 CLEC4G VIPR1 LHX2 DHODH PTH1R

184 DEGs were identified from four profile datasets, including 92 upregulated genes and 92 downregulated genes in the hepatocellular carcinoma tissues, compared to normal liver tissues. The upregulated genes were listed from the largest to the smallest of fold changes, and downregulated genes were listed from the smallest to largest of fold changes. DEGs, differentially expressed genes.

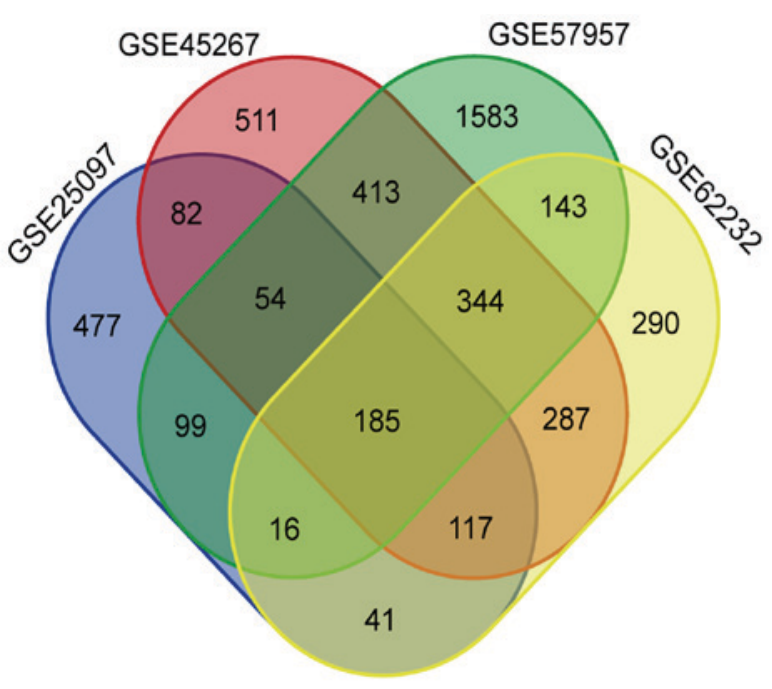

Figure 1. Identification of 185 commonly changed DEGs from the four cohort profile data sets (GSE25097, GSE45267, GSE57957 and GSE62232). Different color areas represent different datasets. The cross areas indicate the commonly changed DEGs. DEGs were identified with classical t-test; statistically significant DEGs were defined with $\mathrm{P}<0.01$ and $[\log \mathrm{FC}]>1$ as the cut-off criteria. DEGs, differentially expressed genes.

the predominant group among upregulated genes, followed by ATP binding (25\%) and DNA binding (18.5\%), while the downregulated genes were mainly signed to the calcium ion binding and serine-type endopeptidase activity groups. For the cellular component group, upregulated genes were mainly enriched in the nucleoplasm, nucleus, and cytoplasm, and the downregulated genes were mainly enriched in the extracellular region, extracellular space, and extracellular exosome.
Signaling pathway enrichment analysis. To investigate functional and signaling pathway enrichment of the gene signatures, we performed a pathway analysis using online websites of DAVID and KEGG (http://www.genome. $\mathrm{jp} / \mathrm{kegg} /$ ) pathways and GO. The upregulated DEGs were mainly enriched in DNA replication, cell cycle, HTLV-I infection, oocyte meiosis, and p53 signaling pathway, while the downregulated DEGs were mainly enriched in metabolic pathways, caffeine metabolism, mineral absorption, thyroid hormone signaling pathway, and AMPK signaling pathway (Table III, Fig. 3).

Key candidate genes and pathways identification with DEGs protein-protein interaction network (PPI) and modular analysis. Based on the analysis in the STRING database (Available online: http://string-db.org) (20) and Cytoscape software (17), relevant protein-protein interactions (PPI) were obtained and visualized, containing 184 nodes and 1166 edges (Fig. 4A). After a topological analysis, 15 genes with a node degree $\geq 36$ (i.e., each node had more than 10 connections/interactions) were selected as representative hub genes. The most significant 15 node degree genes were TOP2A, PCNA, CCNB2, AURKA, CDKN3, BUB1, RFC4, CEP55, DLGAP5, MCM2, PRC1, RACGAP1, TPX2, CDC20, and MCM4. Based on the degree of importance, one significant module was chosen for further analysis through Cytotype MCODE from the PPI network complex. Pathway enrichment analysis showed that Module 1 consisted of 33 nodes and 507 edges (Fig. 4B, Table IV), which are mainly associated with DNA replication, cell cycle, switching of origins to a post-replicative state, and activation of ATR in response to replication stress by pathway enrichment analysis. 

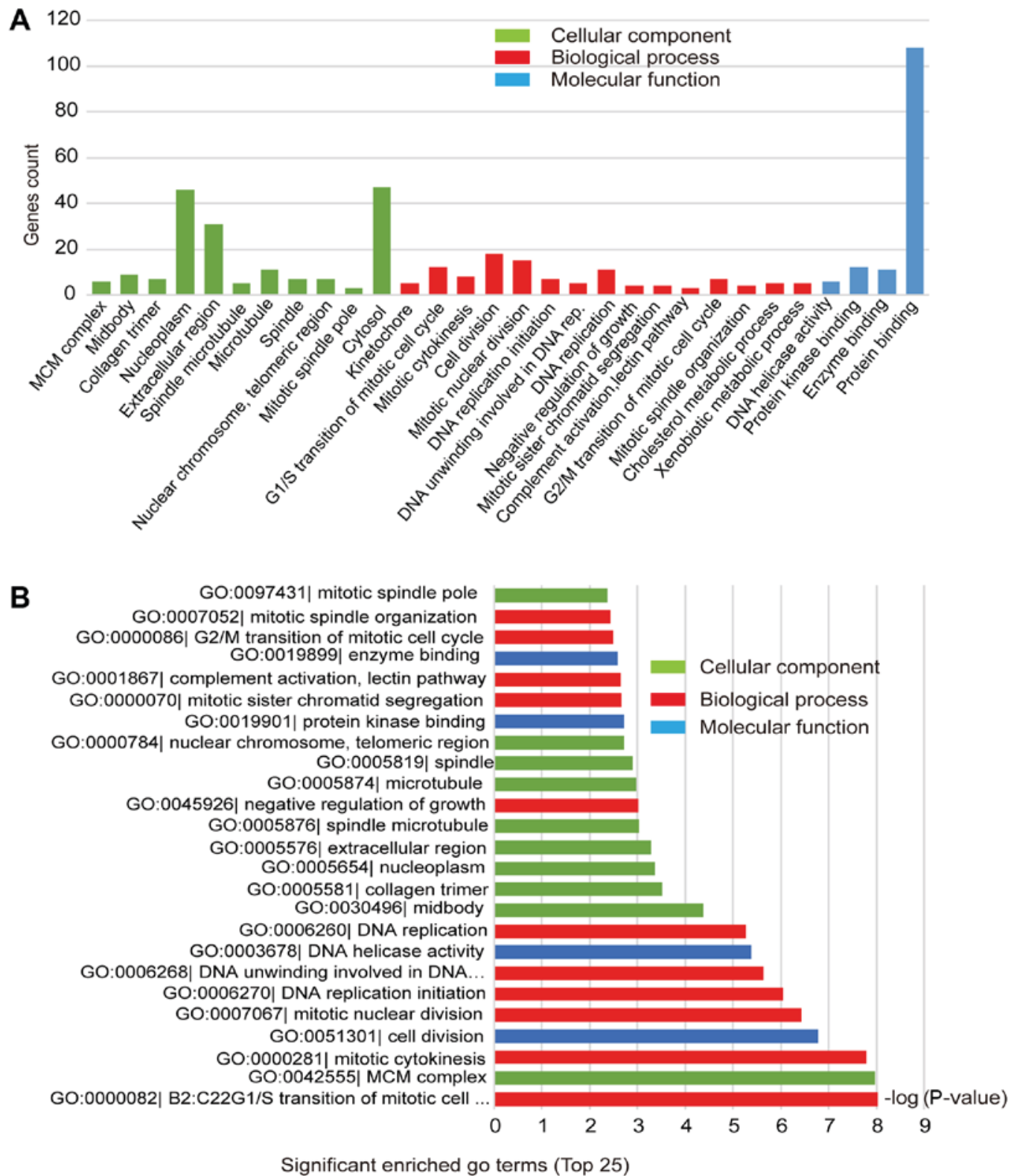

Figure 2. GO analysis and significantly enriched GO in terms of DEGs in HCC. (A) GO analysis classified the DEGs into three groups (molecular function, biological process and cellular component). (B) Significant Enriched GO Terms of DEGs in HCC based on their functions. GO, gene ontology; DEG, differentially expressed gene; HCC, hepatocellular carcinoma.

Validation of the DEGs in cBio portal dataset. To further confirm the validity of the identified DEGs, cBio portal, an online integrated data mining system, was used to analyze the genetic alteration of genes. Among the four HCC studies analyzed $(12,21,22)$, alterations ranging from 15.2 to $68.9 \%$ were found including the gene sets submitted for analysis (Fig. 5A). The most pronounced genomic changes observed across samples from the TCGA study was presented using OncoPrint. Two genes, UBE2T and CCNE2, with the largest mutation frequency found based on the TCGA database in HCC were also identified as the hub genes in this study. The results showed low mutation frequency of the three hub genes-TOP2A, PCNA, and AURKA in HCC samples-investigated in the present study (Fig. 5B), but in the TCGA breast cancer, the three genes showed high alteration frequency $(23,24)$ (Fig. 5C). Thus, the roles of TOP2A, PCNA, and AURKA as biomarkers in HCC progression and histological grading should be investigated more systematically.

\section{Discussion}

HCC is one of the most common malignant neoplasms worldwide. Advanced-stage HCC is notoriously difficult to treat. Although various causes and underlying mechanisms of HCC formation and progression have been revealed by several basic and clinical studies in the past several decades, the worldwide incidence and mortality of HCC is still very high. Different from a single genetic or cohort study, this study analyzed four original microarray datasets, including $436 \mathrm{HCC}$ and 94 normal liver tissues. In total, 185 DEGs were identified in HCC, including 92 upregulated genes and 92 downregulated genes. GO was performed, which showed that the upregulated DEGs 
Table II. The significant enriched analysis of DEGs in hepatocellular carcinoma.

A, Upregulated

\begin{tabular}{llrr}
\hline Term & \multicolumn{1}{c}{ Description } & Count & P-value \\
\hline GO:0051301 & Cell division & 18 & $2.36 \times 10^{-12}$ \\
GO:0007067 & Mitotic nuclear division & 15 & $3.38 \times 10^{-11}$ \\
GO:0000281 & Mitotic cytokinesis & 8 & $1.12 \times 10^{-10}$ \\
GO:0000082 & G1/S transition of mitotic cell cycle & 11 & $1.18 \times 10^{-10}$ \\
GO:0042555 & MCM complex & 6 & $2.90 \times 10^{-10}$ \\
GO:0005654 & Nucleoplasm & 37 & $7.64 \times 10^{-09}$ \\
GO:0006270 & DNA replication initiation & 6 & $1.32 \times 10^{-08}$ \\
GO:0003678 & DNA helicase activity & 5 & $1.27 \times 10^{-07}$ \\
GO:0006268 & DNA unwinding involved in DNA replication & 9 & $1.38 \times 10^{-07}$ \\
GO:0030496 & Midbody & 9 & $2.03 \times 10^{-07}$ \\
GO:0006260 & DNA replication & 23 & $1.39 \times 10^{-06}$ \\
GO:0005524 & ATP binding & 35 & $4.82 \times 10^{-06}$ \\
GO:0005829 & Cytosol & 56 & $1.16 \times 10^{-06}$ \\
GO:0005515 & Protein binding & 10 & $1.92 \times 10^{-05}$ \\
GO:0005874 & Microtubule & & \\
\hline
\end{tabular}

B, Downregulated

\begin{tabular}{llrr}
\hline Term & \multicolumn{1}{c}{ Description } & Count & P-value \\
\hline GO:0005576 & Extracellular region & 28 & $8.07 \times 10^{-09}$ \\
GO:0005615 & Extracellular space & 19 & $8.81 \times 10^{-05}$ \\
GO:0005581 & Collagen trimer & 6 & $9.21 \times 10^{-05}$ \\
GO:0004252 & Serine-type endopeptidase activity & $3.22 \times 10^{-04}$ \\
GO:0001867 & Complement activation, lectin pathway & 3 & $5.48 \times 10^{-04}$ \\
GO:0071276 & Cellular response to cadmium ion & 3 & 0.003 \\
GO:0071294 & Cellular response to zinc ion & 3 & 0.004 \\
GO:0045926 & Negative regulation of growth & 3 & 9.004 \\
GO:0006508 & Proteolysis & 3 & 0.004 \\
GO:0034364 & High-density lipoprotein particle & 3 & 0.005 \\
\hline
\end{tabular}

DEGs, differentially expressed genes; GO, gene ontology.

were mainly enriched in cell division, mitotic nuclear division, mitotic cytokinesis, and G1/S transition of the mitotic cell cycle. Pathway enrichment was analyzed based on the KEGG database to assess the functional relevance of DEGs. On the basis of the analysis in the STRING database and Cytoscape software, relevant PPIs were obtained and visualized that contained 184 nodes and 1166 edges. The most significant 15 hub genes were selected, including TOP2A, PCNA, CCNB2, AURKA, CDKN3, BUB1, RFC4, CEP55, DLGAP5, MCM2, PRC1, RACGAP1, TPX2, CDC20, and MCM4.

Cell cycle-related gene TOP2A (DNA topoisomerase II alpha) encodes a DNA topoisomerase, an enzyme that controls and alters the topologic states of DNA during transcription. In many cancers including breast, ovarian, colon, and small-cell lung cancers, TOP2A has also been shown as a valuable prognostic marker for tumor grading, recurrences, and a predictor of poorer patient survival (25-27). In this study,
TOP2A is the top degree hub gene and overexpressed in tumor tissue compared to adjacent non-tumoral or normal tissue. Consistent with our studies, Wong et al (28), used microarrays to examine 22 HCC cell lines; their array-based transcriptional mapping results showed that TOP2A was upregulated in 14/22 cell lines through DNA copy gains. Furthermore, TMA analysis of 172 liver tumors showed that TOP2A expressions significantly correlated with advanced histological grading, microvascular invasion, aggressive biological behavior, and chemotherapy resistance. High-grade TOP2A expressions showed significantly lower overall survival. Thus, TOP2A as a biomarker in histological grading and a target in chemotherapy resistance should be investigated more systematically. The second hub gene PCNA, proliferating cell nuclear antigen, encodes the protein which is found in the nucleus and is a cofactor of DNA polymerase delta and involved in the RAD6-dependent DNA repair pathway in response to DNA 


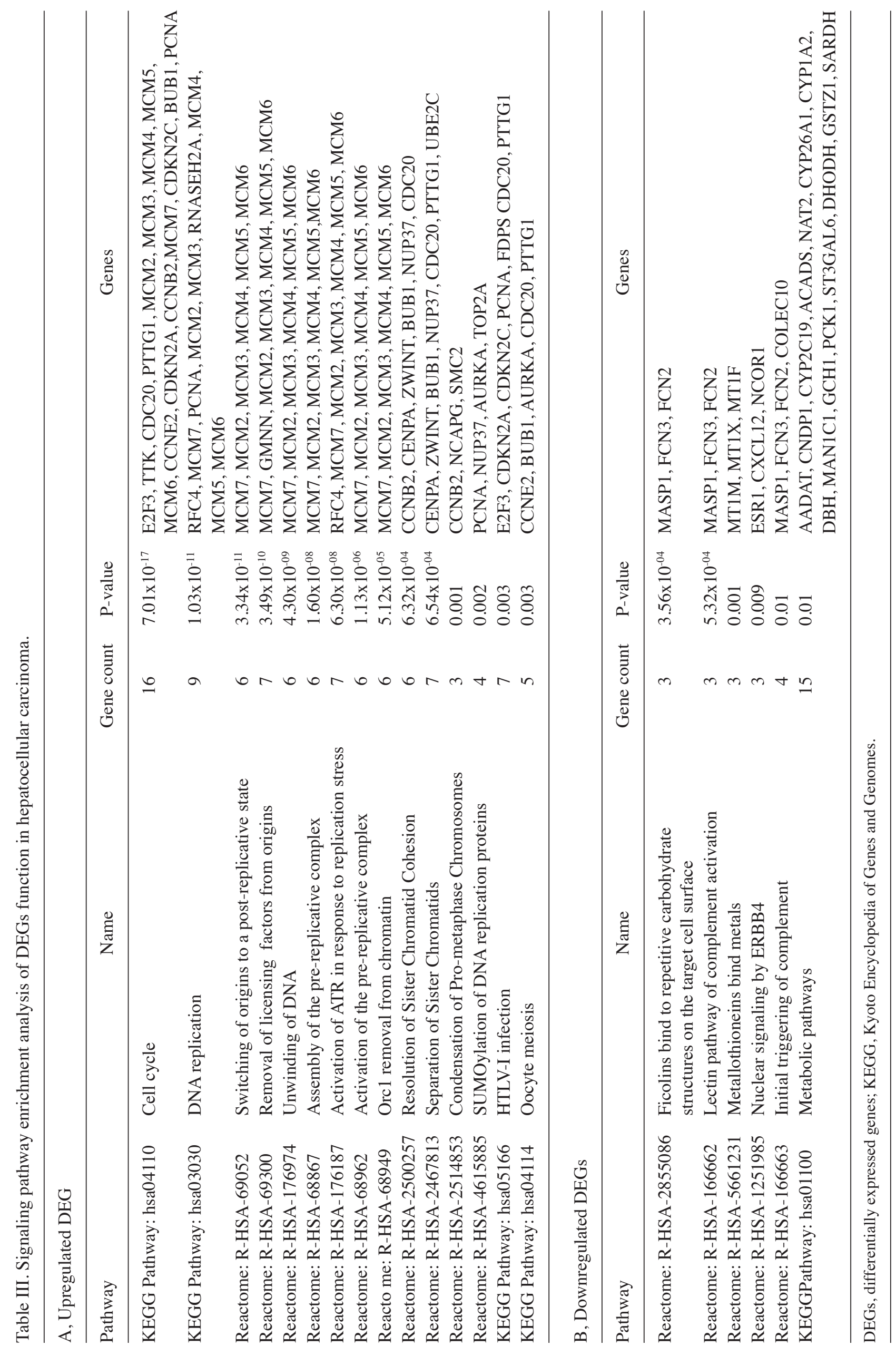


Table IV. Pathway enrichment analysis of Module 1 genes function.

\begin{tabular}{llrr}
\hline Term & & \multicolumn{1}{c}{ Description } & Count \\
\hline KEGG:hsa04110 & Cell cycle & 11 & $6.64 \times 10^{-15}$ \\
KEGG:hsa03030 & DNA replication & 8 & $3.50 \times 10^{-13}$ \\
R-HSA-69052 & Switching of origins to a post-replicative state & 6 & $3.93 \times 10^{-13}$ \\
R-HSA-176974 & Unwinding of DNA & 6 & $5.14 \times 10^{-11}$ \\
R-HSA-68867 & Assembly of the pre-replicative complex & 6 & $1.94 \times 10^{-10}$ \\
R-HSA-176187 & Activation of ATR in response to replication stress & 7 & $2.88 \times 10^{-10}$ \\
R-HSA-69300 & Removal of licensing factors from origins & 6 & $3.98 \times 10^{-10}$ \\
R-HSA-68962 & Activation of the pre-replicative complex & 6 & $1.26 \times 10^{-08}$ \\
R-HSA-68949 & Orc1 removal from chromatin & 6 & $7.66 \times 10^{-07}$ \\
R-HSA-2467813 & Separation of Sister Chromatids & 6 & $8.33 \times 10^{-05}$ \\
R-HSA-2500257 & Resolution of Sister Chromatid Cohesion & 5 & $2.19 \times 10^{-04}$ \\
R-HSA-174178 & APC/C:Cdh1 mediated degradation of Cdc20 and other & 4 & $7.57 \times 10^{-04}$ \\
KEGG:hsa04114 & APC/C:Cdh1 targeted proteins in late mitosis/early G1 & 4 & 0.001 \\
R-HSA-179409 & Oocyte meiosis & 3 & 0.00179182
\end{tabular}

KEGG, Kyoto Encyclopedia of Genes and Genomes.

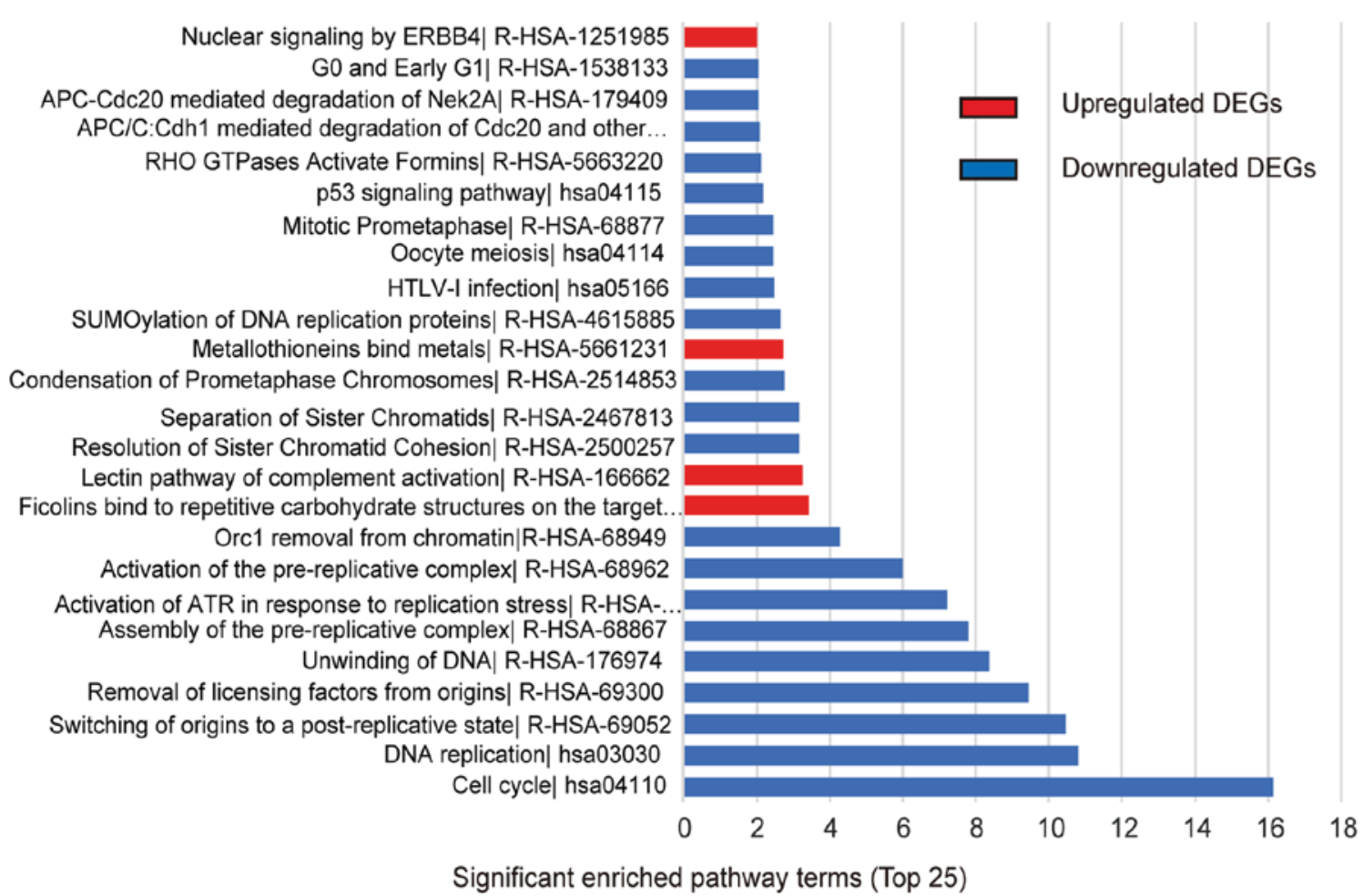

Figure 3. Significantly enriched pathway in terms of DEGs in colorectal cancer. DEGs functional and signaling pathway enrichment were conducted using online websites of KEGG PATHWAY, Reactomen, and Gene Ontology analysis. DEGs, differentially expressed genes; KEGG, Kyoto Encyclopedia of Genes and Genomes.

damage. Owing to its function in cell proliferation, PCNA plays an important role in cancer progression (29). Combined hepatocellular-cholangiocarcinoma $(\mathrm{CHC})$ is a malignant subtype of primary liver tumor containing elements of both HCC and intrahepatic cholangiocarcinoma (ICC). In patients who underwent hepatectomy with curative intent, nontumor ductular reactions (DRs) produced by hepatic progenitor cells (HPCs) in nontumor liver tissue were an independent prognostic indicator for disease-free survival (DFS) and overall survival (OS). PCNA could label the index of the ductular reaction (PI-DR). A higher level of PI-DR contributes to intratumoral HPC activation (30), fibrosis, hepatocyte replication, and hepatic inflammation which is predictive of a high recurrence rate. Moreover, increased PI-DR expression 

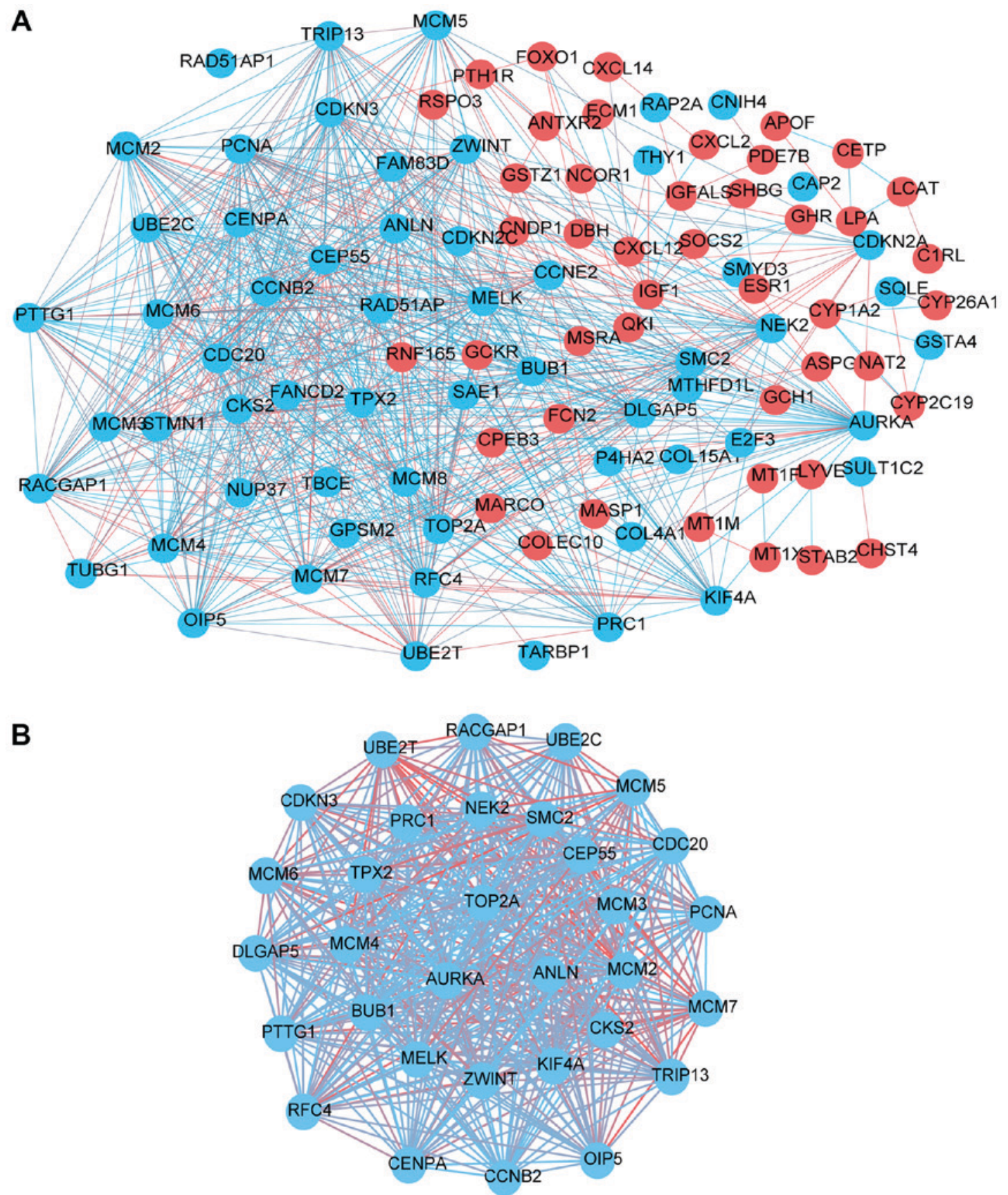

Figure 4. DEGs PPI network complex and modular analysis. (A) Using the STRING online database, total of 184 DEGs (92 upregulated in blue standing for upregulation and 92 downregulated genes in red standing for downregulation) were filtered into the DEGs PPI network complex. (B) The most significant module consists of 33 nodes and 507 edges, which are mainly associated with DNA replication, cell cycle, switching of origins to a post-replicative state, and activation of ATR in response to replication stress. DEGs, differentially expressed genes; PPI, protein-protein interaction.

was also associated with multicentric occurrence (MO) and microvascular invasion (MVI) of HCC (31). However, several studies found conflicting results, which showed that PCNA had little prognostic value in tumor tissue $(32,33)$. Because one part of PCNA trimers was not engaged in DNA synthesis (34). Similar to the PCNA function in maintaining the stability of chromosomal replication and segregation in cellular mitosis, another gene-aurora kinase A (AURKA)-was found upregulated in our experiment. AURKA is a cell cycle regulated kinase that appears to be involved in microtubule formation. A clinical trial by Jeng et al (35), showed that AURKA was overexpressed in 137 (61\%) of 223 patients with HCC. AURKA overexpression coincided with portal vein tumor invasion, regardless of tumor size. Furthermore, AURKA was found involved in p53 signaling pathway and interacted directly with p53 mutation (36). Aurora-A and p53 mutation had a synergistic effect promoting tumor progression and poor prognosis (35). In p53-altered (deleted or mutated) liver cancer, the tumor suppressor protein $\mathrm{p} 19^{\mathrm{ARF}}$ was activated and mediated $\mathrm{G} 2 / \mathrm{M}$ cell cycle arrest. MYC, a proto-oncogene, was overexpressed and directly bound to AURKA, which stabilized MYC to overcome G2 to $\mathrm{M}$ cell cycle arrest and promote tumor cell survival. A previous study found conformation-changing AURKA inhibitors could prevent formation of MYC-AURKA 
A

Summary of alterations for up-regulated DEGs in hepatocellular carcinoma (4 studies/ DEGs)

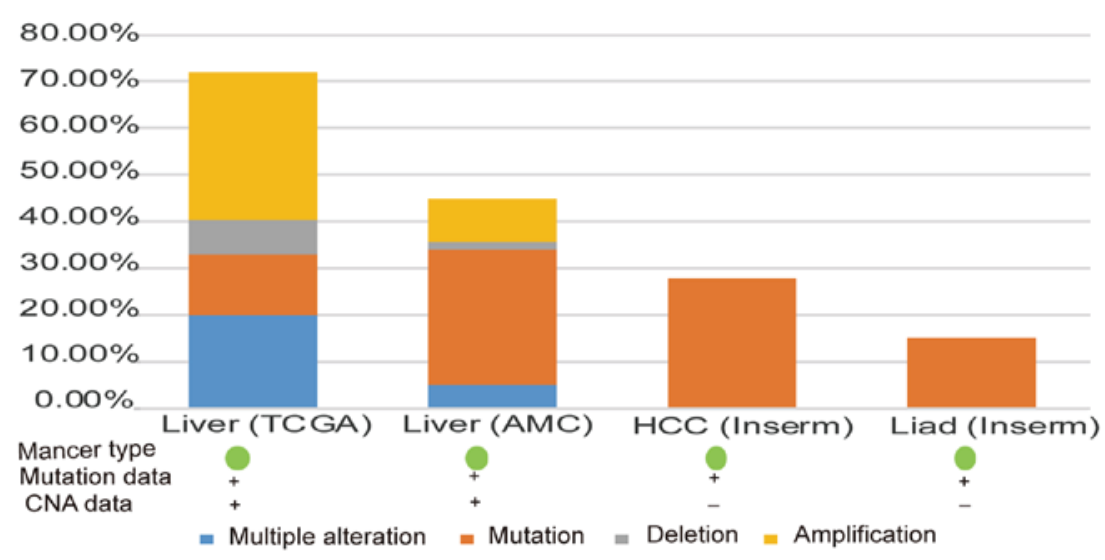

B TCGA hepatocellular carcinoma with sequencing and CAN data (442 samples)/5 Genes

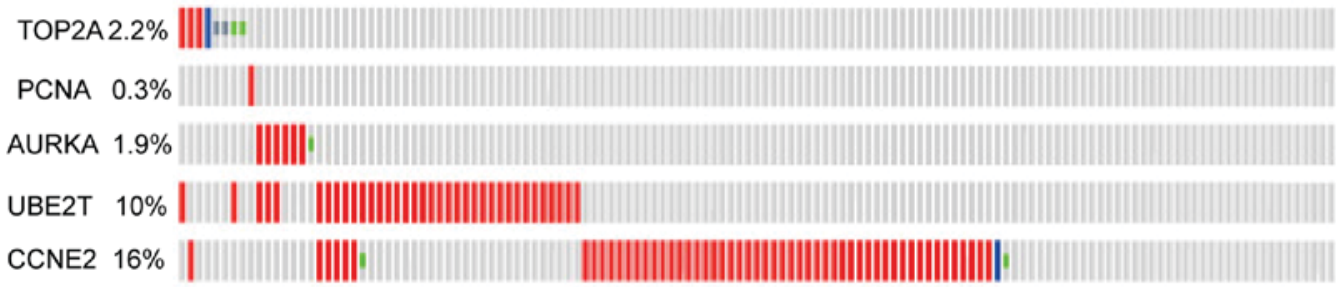

$\begin{aligned} \text { Genetic alteration } & \text { Amplification Deep deleption }=\text { Truncating mutation (putative passenger) } \\ & \text { Missense mutation (putative passenger) }\end{aligned}$

Case Set: Tumors samples with sequencing and CAN data (366 patients/366 samples)

Altered in $265(72 \%)$ of 366 sequenced cases/patients (366 total)

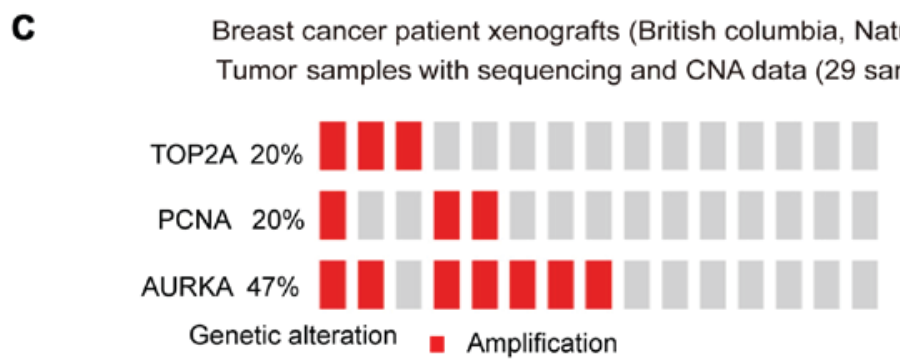

Case Set: Tumor samples with sequencing and CNA data (15 patients / 29 samples)

Altered in $8(53 \%)$ of 15 sequenced cases/patients ( 15 total)

Figure 5. Validation of the DEGs in cBio portal Dataset. (A) Overview of changes in the identified DEGs in genomics data sets available in 4 different HCC studies. (B) OncoPrint: A visual summary of alteration across TCGA hepatocellular carcinoma based on a query of DEGs. (C) OncoPrint, A visual summary of alteration across TCGA breast cancer based on a query of the three genes-TOP2A, PCNA, and AURKA. Distinct genomic alterations including mutations and CNAs (exemplified by gene amplifications and homozygous deletions) are summarized and color coded presented by $\%$ changes in particular affected genes in individual tumor samples. Each row represents a gene, and each column represents a tumor sample. Red bars designate gene amplifications, blue bars represent homozygous deletions, and green squares indicate nonsynonymous mutations. DEGs, differentially expressed genes; HCC, hepatocellular carcinoma; CNS, copy number alterations; TCGA, The Cancer Genome Atlas.

complexes and hence, degradation of MYC (37). Therefore, therapeutic strategy targeting MYC-AURKA complexes could be considered for this subtype of HCCs.

Besides the cycle-related pathways and p53 signaling, $\mathrm{T}$ lymphotropic virus type I (HTLV-I) infection-related biological processes and pathways were also identified in our study. HTLV-1 infection, especially combined with HCV infection, increased HCC mortality (38). Tax, an HTLV-I oncoprotein, contributes to chromosome aneuploidy, cytokinesis failure, and multinucleated cells primarily by directly binding and activating the $\mathrm{CDC} 20$-associated anaphase promoting complex $\left(\mathrm{APC}^{\mathrm{CDC} 20}\right.$ ) during $\mathrm{S}$ phase to delay mitotic progression and faulty mitosis (39). Other DEGs in our study associated with HTLV-I infection-related pathway were FDPS, CDC20, E2F, CDKN2A, CDK2N2C, PTTG1, and PCNA.

Consistent with our studies, Jin et al (40), analyzed gene expression profiles GSE6222, GSE41804, and GSE51401 that contained 117 samples, including 54 cases and 63 controls 
from which 1347 DEGs were identified, including 2920 upregulated genes and 2231 downregulated genes. The top 10 hug genes were SPINK1, TOP2A, ASPM, GPC3, ANLN, SULT1C2, CCNB1, PEG10, CDKN3, and ECT2. The main pathway of the identified DEGs were those involved in cell cycle and oocyte meiosis, which were also identified in our study. However, Jin's study was based on a dataset only generated from Chinese and Japanese patients. Different from Yin's report, we analyzed four datasets generated from American, Chinese, Singaporean, and French patients, thereby representing different populations. Apart from cell cycle-related pathways, HTLV-I infection and p53 signaling pathway were identified, because the incidence of NAFLD and steatohepatitis was high in European and American patients $(41,42)$.

HCC is a group of complex and heterogeneous tumors. The mechanism mainly involves chromosomal and microsatellite instability (43), and the later involves the inactivation or mutation of DNA mismatch repair genes. Our study found chromosomal instability-related genes including BUB, PCNA, CDC20, and AURKA. The characteristics of HCC cannot be explained only by analysis of gene expression profiles, although gene expression profiles could reveal some of the underlying mechanism in cancer progression. Various factors should be explored, including gene mutation, methylation, miRNA, and lncRNA, which could likely participate in HCC carcinogenesis and chemotherapy resistance. For instance, TSLNC8, a long intergenic noncoding RNA on chromosome 8p12, was characterized as a novel tumor suppressor by modulating the IL-6/STAT3 signaling pathway and being inversely correlated with HCC embolus, nodules, and differentiation stage (44). A clinical trial based on 2079 cirrhotic patients with long term follow-up found that the incidence of HCC induced by the etiology of cirrhosis was different. Chronic viral hepatitis patients had a higher cumulative risk of HCC than those with primary biliary cirrhosis and NAFLD, while those with autoimmune liver diseases (AIH) had the lowest risk (45).

Taken together, in our study we have identified 185 DEG candidate genes using integrated bioinformatical analysis, and found 15 mostly changed hub genes, which were significantly enriched in cell cycle process, DNA replication, p53 signaling, and HTLV-I infection-related biological processes and pathways. These findings could promote our understanding of the cause and molecular mechanisms underlying the development of HCC, and these candidate and signal pathways could be the targets of clinical therapy for HCC.

\section{Acknowledgements}

Not applicable.

\section{Funding}

This study was supported by the project of 'Medical Professionals Cross Fund', Shanghai Jiao Tong University, Shanghai, China (grant no. YG2013MS01), the 863 Program (grant nos. 2012AA02A515 and 2012AA021802), the National Nature Science Foundation of China (grant nos. 81421061, 81273596, J1210047, 30900799, 81361120389 and 30972823) and the National key research and development program (grant nos. 2016YFC0905000, 2016YFC1200200 and 2016YFC0906400).

\section{Availability of data and materials}

The datasets generated and analyzed during the current study are available from the corresponding author on reasonable request.

\section{Authors' contributions}

TX was involved in drafting the manuscript, design of the work, acquisition and analysis of data, and approval of the final version to be published. TY contributed to conception and design of the study, and the acquisition of the data. QZ was responsible for analysis and interpretation of the data. All authors read and approved the final manuscript.

\section{Ethics approval and consent to participate}

Not applicable.

\section{Consent for publication}

Not applicable.

\section{Competing interests}

The authors declare that they have no competing interests.

\section{References}

1. Slotta JE, Kollmar O, Ellenrieder V, Ghadimi BM and Homayounfar K: Hepatocellular carcinoma: Surgeon's view on latest findings and future perspectives. World J Hepatol 7: $1168-1183,2015$

2. Chen W, Zheng R, Baade PD, Zhang S, Zeng H, Bray F, Jemal A, $\mathrm{Yu}$ XQ and He J: Cancer statistics in China, 2015. CA Cancer J Clin 66: 115-132, 2016.

3. Nair S, Mason A, Eason J, Loss G and Perrillo RP: Is obesity an independent risk factor for hepatocellular carcinoma in cirrhosis? Hepatology 36: 150-155, 2002.

4. Baffy G, Brunt EM and Caldwell SH: Hepatocellular carcinoma in non-alcoholic fatty liver disease: An emerging menace. J Hepatol 56: 1384-1391, 2012.

5. Yang JD and Roberts LR: Hepatocellular carcinoma: A global view. Nat Rev Gastroenterol Hepatol 7: 448-458, 2010.

6. Valle JW, Dangoor A, Beech J, Sherlock DJ, Lee SM, Scarffe JH, Swindell R and Ranson M: Treatment of inoperable hepatocellular carcinoma with pegylated liposomal doxorubicin (PLD): Results of a phase II study. Br J Cancer 92: 628-630, 2005.

7. Llovet JM and Bruix J: Molecular targeted therapies in hepatocellular carcinoma. Hepatology 48: 1312-1327, 2008

8. Whittaker S, Marais R and Zhu AX: The role of signaling pathways in the development and treatment of hepatocellular carcinoma. Oncogene 29: 4989-5005, 2010.

9. Wang XW, Hussain SP, Huo TI, Wu CG, Forgues M, Hofseth LJ, Brechot $\mathrm{C}$ and Harris CC: Molecular pathogenesis of human hepatocellular carcinoma. Toxicology 181-182: 43-47, 2002.

10. Rinninella E, Zocco MA, De Gaetano A, Iezzi R, Campanale M, Cesario V, Barbaro F, Ponziani FR, Caracciolo G, Triarico S, et al: From small nodule to overt HCC: A multistep process of carcinogenesis as seen during surveillance. Eur Rev Med Pharmacol Sci 16: 1292-1294, 2012.

11. Brazma A, Parkinson H, Sarkans U, Shojatalab M, Vilo J, Abeygunawardena N, Holloway E, Kapushesky M, Kemmeren P, Lara GG, et al: ArrayExpress-a public repository for microarray gene expression data at the EBI. Nucleic Acids Res 31: 68-71, 2003. 
12. Schulze K, Imbeaud S, Letouze E, Alexandrov LB, Calderaro J, Rebouissou S, Couchy G, Meiller C, Shinde J, Soysouvanh F, et al: Exome sequencing of hepatocellular carcinomas identifies new mutational signatures and potential therapeutic targets. Nat Genet 47: 505-511, 2015.

13. Roessler S, Jia HL, Budhu A, Forgues M, Ye QH, Lee JS, Thorgeirsson SS, Sun Z, Tang ZY, Qin LX and Wang XW: A unique metastasis gene signature enables prediction of tumor relapse in early-stage hepatocellular carcinoma patients. Cancer Res 70: 10202-10212, 2010.

14. Tung EK, Mak CK, Fatima S, Lo RC, Zhao H, Zhang C, Dai H, Poon RT, Yuen MF, Lai CL, et al: Clinicopathological and prognostic significance of serum and tissue Dickkopf-1 levels in human hepatocellular carcinoma. Liver Int 31: 1494-1504, 2011.

15. Ashburner M, Ball CA, Blake JA, Botstein D, Butler H, Cherry JM, Davis AP, Dolinski K, Dwight SS, Eppig JT, et al: Gene ontology: Tool for the unification of biology. The gene ontology consortium. Nat Genet 25: 25-29, 2000 .

16. Lebrec JJ, Huizinga TW, Toes RE, Houwing-Duistermaat JJ and van Houwelingen HC: Integration of gene ontology pathways with North American Rheumatoid Arthritis Consortium genome-wide association data via linear modeling. BMC Proc 3 (Suppl 7): S94, 2009.

17. Shannon P, Markiel A, Ozier O, Baliga NS, Wang JT, Ramage D, Amin N, Schwikowski B and Ideker T: Cytoscape: A software environment for integrated models of biomolecular interaction networks. Genome Res 13: 2498-2504, 2003.

18. Sung WK, Zheng H, Li S, Chen R, Liu X, Li Y, Lee NP, Lee WH, Ariyaratne PN, Tennakoon C, et al: Genome-wide survey of recurrent HBV integration in hepatocellular carcinoma. Nat Genet 44: 765-769, 2012.

19. Mah WC, Thurnherr T, Chow PK, Chung AY, Ooi LL, Toh HC, Teh BT, Saunthararajah Y and Lee CG: Methylation profiles reveal distinct subgroup of hepatocellular carcinoma patients with poor prognosis. PLoS One 9: e104158, 2014.

20. Franceschini A, Szklarczyk D, Frankild S, Kuhn M, Simonovic M Roth A, Lin J, Minguez P, Bork P, von Mering C and Jensen LJ: STRING v9.1: Protein-protein interaction networks, with increased coverage and integration. Nucleic Acids Res 41: D808-D815, 2013.

21. Pilati C, Letouze E, Nault JC, Imbeaud S, Boulai A, Calderaro J, Poussin K, Franconi A, Couchy G, Morcrette G, et al: Genomic profiling of hepatocellular adenomas reveals recurrent FRK-activating mutations and the mechanisms of malignant transformation. Cancer Cell 25: 428-441, 2014

22. Ahn SM, Jang SJ, Shim JH, Kim D, Hong SM, Sung CO, Baek D, Haq F, Ansari AA, Lee SY, et al: Genomic portrait of resectable hepatocellular carcinomas: Implications of RB1 and FGF19 aberrations for patient stratification. Hepatology 60: 1972-1982, 2014.

23. Ejlertsen B, Tuxen MK, Jakobsen EH, Jensen MB, Knoop AS Højris I, Ewertz M, Balslev E, Danø H, Vestlev PM, et al: Adjuvant cyclophosphamide and docetaxel with or without epirubicin for early TOP2A-normal breast cancer: DBCG 07-READ, an open-label, phase III, randomized trial. J Clin Oncol 35: 2639-2646, 2017.

24. Di Leo A, Desmedt C, Bartlett JM, Piette F, Ejlertsen B, Pritchard KI, Larsimont D, Poole C, Isola J, Earl H, et al: HER2 and TOP2A as predictive markers for anthracycline-containing chemotherapy regimens as adjuvant treatment of breast cancer: A meta-analysis of individual patient data. Lancet Oncol 12: 1134-1142,2011.

25. Fritz P, Cabrera CM, Dippon J, Gerteis A, Simon W, Aulitzky WE and van der Kuip H: c-erbB2 and topoisomerase IIalpha protein expression independently predict poor survival in primary human breast cancer: A retrospective study. Breast Cancer Res 7: R374-R384, 2005.

26. Lazaris AC, Kavantzas NG, Zorzos HS, Tsavaris NV and Davaris PS: Markers of drug resistance in relapsing colon cancer. J Cancer Res Clin Oncol 128: 114-118, 2002.

27. Costa MJ,Hansen CL, Holden JA and Guinee D Jr: Topoisomerase II alpha: Prognostic predictor and cell cycle marker in surface epithelial neoplasms of the ovary and peritoneum. Int J Gynecol Pathol 19: 248-257, 2000.

28. Wong N, Yeo W, Wong WL, Wong NL, Chan KY, Mo FK, Koh J, Chan SL, Chan AT, Lai PB, et al: TOP2A overexpression in hepatocellular carcinoma correlates with early age onset, shorter patients survival and chemoresistance. Int J Cancer 124: 644-652, 2009
29. Stoimenov I and Helleday T: PCNA on the crossroad of cancer. Biochem Soc Trans 37: 605-613, 2009.

30. Yoon SM, Gerasimidou D, Kuwahara R, Hytiroglou P, Yoo JE, Park YN and Theise ND: Epithelial cell adhesion molecule (EpCAM) marks hepatocytes newly derived from stem/progenitor cells in humans. Hepatology 53: 964-973, 2011.

31. Cai X, Zhai J, Kaplan DE, Zhang Y, Zhou L, Chen X, Qian G, Zhao Q, Li Y, Gao L, et al: Background progenitor activation is associated with recurrence after hepatectomy of combined hepatocellular-cholangiocarcinoma. Hepatology 56: 1804-1816, 2012.

32. Visakorpi T: Proliferative activity determined by DNA flow cytometry and proliferating cell nuclear antigen (PCNA) immunohistochemistry as a prognostic factor in prostatic carcinoma. J Pathol 168: 7-13, 1992

33. Dworakowska D, Gozdz S, Jassem E, Badzio A, Kobierska G, Urbaniak A, Skokowski J, Damps I and Jassem J: Prognostic relevance of proliferating cell nuclear antigen and p53 expression in non-small cell lung cancer. Lung Cancer 35: 35-41, 2002.

34. Wang SC, Nakajima Y, Yu YL, Xia W, Chen CT, Yang CC, McIntush EW, Li LY, Hawke DH, Kobayashi R and Hung MC: Tyrosine phosphorylation controls PCNA function through protein stability. Nat Cell Biol 8: 1359-1368, 2006.

35. Jeng YM, Peng SY, Lin CY and Hsu HC: Overexpression and amplification of Aurora-A in hepatocellular carcinoma. Clin Cancer Res 10: 2065-2071, 2004

36. Chen SS, Chang PC, Cheng YW, Tang FM and Lin YS Suppression of the STK15 oncogenic activity requires a transactivation-independent p53 function. EMBO J 21: 4491-4499, 2002.

37. Dauch D, Rudalska R, Cossa G, Nault JC, Kang TW, Wuestefeld T, Hohmeyer A, Imbeaud S, Yevsa T, Hoenicke L, et al: A MYC-aurora kinase A protein complex represents an actionable drug target in p53-altered liver cancer. Nat Med 22: 744-753, 2016.

38. Tokunaga $\mathrm{M}$, Uto $\mathrm{H}$, Oda $\mathrm{K}$, Tokunaga $\mathrm{M}$, Mawatari $\mathrm{S}$, Kumagai K, Haraguchi K, Oketani M, Ido A, Ohnou N, et al: Influence of human T-lymphotropic virus type 1 coinfection on the development of hepatocellular carcinoma in patients with hepatitis C virus infection. J Gastroenterol 49: $1567-1577,2014$.

39. Liu B, Hong S, Tang Z, Yu H and Giam CZ: HTLV-I Tax directly binds the Cdc20-associated anaphase-promoting complex and activates it ahead of schedule. Proc Natl Acad Sci USA 102: 63-68, 2005.

40. Jin B, Wang W, Du G, Huang GZ Han LT, Tang ZY, Fan DG, $\mathrm{Li} \mathrm{J}$ and Zhang SZ: Identifying hub genes and dysregulated pathways in hepatocellular carcinoma. Eur Rev Med Pharmacol Sci 19: 592-601, 2015

41. Calle EE and Kaaks R: Overweight, obesity and cancer: Epidemiological evidence and proposed mechanisms. Nat Rev Cancer 4: 579-591, 2004

42. White DL, Kanwal F and El-Serag HB: Association between nonalcoholic fatty liver disease and risk for hepatocellular cancer, based on systematic review. Clin Gastroenterol Hepatol 10: 1342-1359.e2, 2012.

43. Lengauer C, Kinzler KW and Vogelstein B: Genetic instabilities in human cancers. Nature 396: 643-649, 1998.

44. Zhang J, Li Z, Liu L, Wang Q, Li S, Chen D, Hu Z, Yu T, Ding J, Li J, et al: Long noncoding RNA TSLNC8 is a tumor suppressor that inactivates the IL-6/STAT3 signaling pathway. Hepatology 67: 171-187, 2017

45. Sharma SA, Kowgier M, Hansen BE, Brouwer WP, Maan R, Wong D, Shah H, Khalili K, Yim C, Heathcote EJ, et al: Toronto HCC risk index: A validated scoring system to predict 10-year risk of HCC in patients with cirrhosis. J hepatol: Aug 24, 2017 doi: 10.1016/j.jhep.2017.07.033.

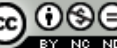

This work is licensed under a Creative Commons Attribution-NonCommercial-NoDerivatives 4.0 International (CC BY-NC-ND 4.0) License. 